\title{
Adult Respiratory Distress Syndrome in Neutropenic Patients
}

MARC D. LAUFE, M.D.

RICHARD H. SIMON, M.D.

ANDREW FLINT, M.D.

JACOB B. KELLER, M.P.H.

Ann Arbor, Michigan
From the Pulmonary and Critical Care Medicine Division, Department of internal Medicine, the Department of Pathology, and the Department of Epidemiology, School of Public Health, University of Michigan Medical Center, Ann Arbor, Michigan. This work was supported in part by the National Heart, Lung, and Blood Institute (HL31963) and by the American Heart Association with funds contributed in part by the American Heart Association of Michigan (84-1058). Requests for reprints should be addressed to Dr. Richard H. Simon, Pulmonary and Critical Care Medicine Division, 3916 Taubman Center, University of Michigan Medical Center, Ann Arbor, Michigan 48109. Manuscript accepted April 10, 1985.
The precise pathophysiologic mechanisms that cause the adult respiratory distress syndrome are unknown. Indirect evidence from human studies and extrapolations from animal models have suggested that phagocytic neutrophils are important in the pathogenesis of this disease. To further evaluate the role of neutrophils, the frequency of neutropenia in 18 bacteremic patients who had the adult respiratory distress syndrome was compared with that in a control group who had bacteremia alone. Three of 18 patients in the group with the adult respiratory distress syndrome were neutropenic as opposed to one of 18 in the control group $(p>0.6)$. Histologic examination of the lungs from two patients with the adult respiratory distress syndrome and neutropenia demonstrated the absence of neutrophlls. It Is Ilkely that there are many pathways that lead to the adult respiratory distress syndrome. Although neutrophils may be involved in some of these processes, this study demonstrates that neutrophils are not required for the development of the syndrome. In the appropriate clinical setting, the diagnosis of the adult respiratory distress syndrome should not be excluded solely because of neutropenia.

The label "adult respiratory distress syndrome" is frequently applied to acute noncardiogenic pulmonary edema [1]. Although the precise mechanisms that lead to the adult respiratory distress syndrome are unknown, it has been suggested that phagocytic neutrophils are a direct cause of the pulmonary damage [2]. The evidence supporting an etiologic role for neutrophils in human adult respiratory distress syndrome is indirect. Increased numbers of neutrophils have been found in the pulmonary capillaries, interstitium, and alveolar spaces [3]. Bronchoalveolar lavage fluid from patients with the syndrome contains increased numbers of neutrophils and increased concentrations of neutrophil secretory products $[4,5]$. In addition, the circulating neutrophils from patients with the adult respiratory distress syndrome possess characteristics suggesting that they have been activated in vivo [6]. Furthermore, stimulated neutrophils are able to kill human cells maintained in tissue culture [7].

In many animal models of the adult respiratory distress syndrome, the lungs can be protected from injury if the animal is made neutropenic prior to administration of the damaging agent [8-12]. To determine if neutrophil depletion also protects the human lung from injury, we examined the effects of neutropenia on patients at risk for the development of the adult respiratory distress syndrome due to bacteremia.

\section{PATIENTS AND METHODS}

A list of patients suspected of having the adult respiratory distress syndrome was compiled by reviewing records maintained by the Respiratory Therapy Department for the period from January 1, 1981, through Decem- 
Figure 1. Serial white blood cell counts in venous blood of Patient 1 demonstrating leukopenia during his hospitalization.

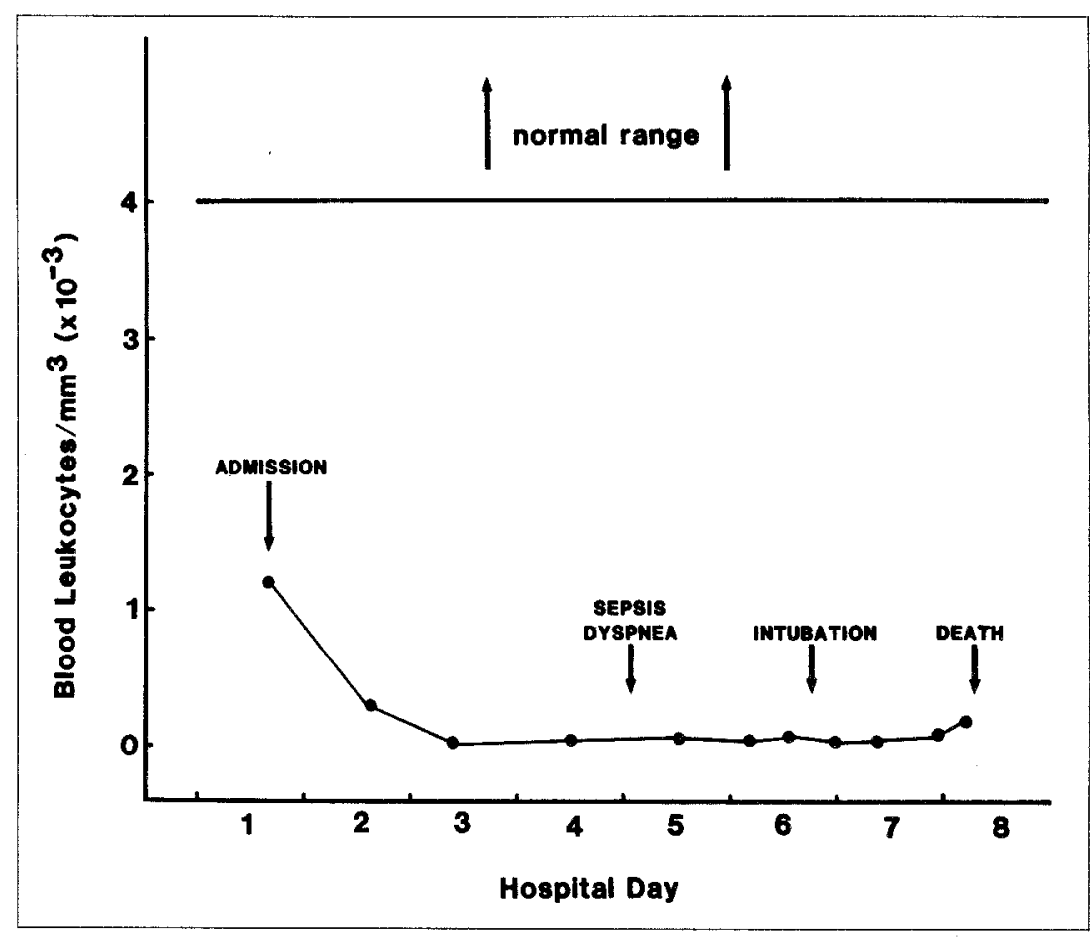

ber 31,1982 . Included in these records were all adults who required assisted mechanical ventilation in the intensive care units at University Hospital. Using computerized records from the microbiology laboratory, we were able to select those patients with respiratory failure who had two or more blood cultures that grew a known bacterial pathogen. After reviewing the available medical records, we identified 18 patients who fulfilled our diagnostic criteria for the adult respiratory distress syndrome and were bacteremic within 48 hours of the development of respiratory symptoms. A patient was considered to have the adult respiratory distress syndrome if all of the following criteria were fulfilled: (1) acute respiratory failure requiring mechanical ventilation, (2) the ratio of partial pressures of oxygen in arterial blood to alveolar gas of 0.2 or less, (3) bilateral pulmonary infiltrates on chest roentgenography, (4) pulmonary capillary wedge pressure of $15 \mathrm{~mm} \mathrm{Hg}$ or less (if available) or absence of clinical evidence of elevated left ventricular end diastolic pressure, and (5) static total respiratory system compliance of $50 \mathrm{ml} / \mathrm{cm} \mathrm{H}_{2} \mathrm{O}$ or less. We hypothesized that if neutropenia protected the lung from injury, then the frequency of neutropenia in the group with the respiratory distress syndrome and bacteremia group should be less than the frequency of neutropenia in bacteremic patients who did not have the syndrome. To test this hypothesis, a control group was chosen by randomly selecting for each patient with the respiratory distress syndrome, a bacteremic patient without respiratory distress who was of the same sex and within three years of the same age. For each patient in both groups, we recorded total leukocyte counts and the percent neutrophils that were present in the peripheral blood at the time of onset of respiratory distress or bacteremia.

\section{RESULTS}

The mean ( \pm SEM) number of neutrophils in peripheral blood for the patients with the respiratory distress syndrome and bacteremia $\left(13,800 \pm 3,800 / \mathrm{mm}^{3}\right)$ did not differ ( $p>0.2$, two-tailed paired $t$ test [13]) from that for the control bacteremic group $\left(9,300 \pm 1,600 / \mathrm{mm}^{3}\right)$. Three of the patients with respiratory distress and bacteremia had neutrophil counts below $1,000 / \mathrm{mm}^{3}(0,12$, and $910 / \mathrm{mm}^{3}$ ), whereas only one patient in the control group was neutropenic $\left(316 / \mathrm{mm}^{3}\right)$. The frequency of neutropenia in the group with the adult respiratory distress syndrome (17 percent) was not statistically different ( $p$ $>0.6$, two-tailed Fisher's exact test [13]) from the frequency of neutropenia in the control group ( 6 percent). By constructing 95 percent one-sided confidence intervals [14], we found that the minimal frequency of neutropenia consistent with the study results for patients with respiratory distress was 5 percent, and the maximal frequency for control patients was 27 percent. Therefore, the possibility of a partial protective effect of neutropenia was not excluded.

Because it has been reported that peripheral blood leukocyte counts in patients with the adult respiratory distress syndrome often decrease abruptly at the onset of lung injury [15], we examined the available serial leukocyte counts from one of the three patients with respiratory distress and neutropenia (Figure 1). The patient (Patient 1) was a 25-year-old man with hepatocellular carcinoma who had received chemotherapy consisting of mitomycin- 

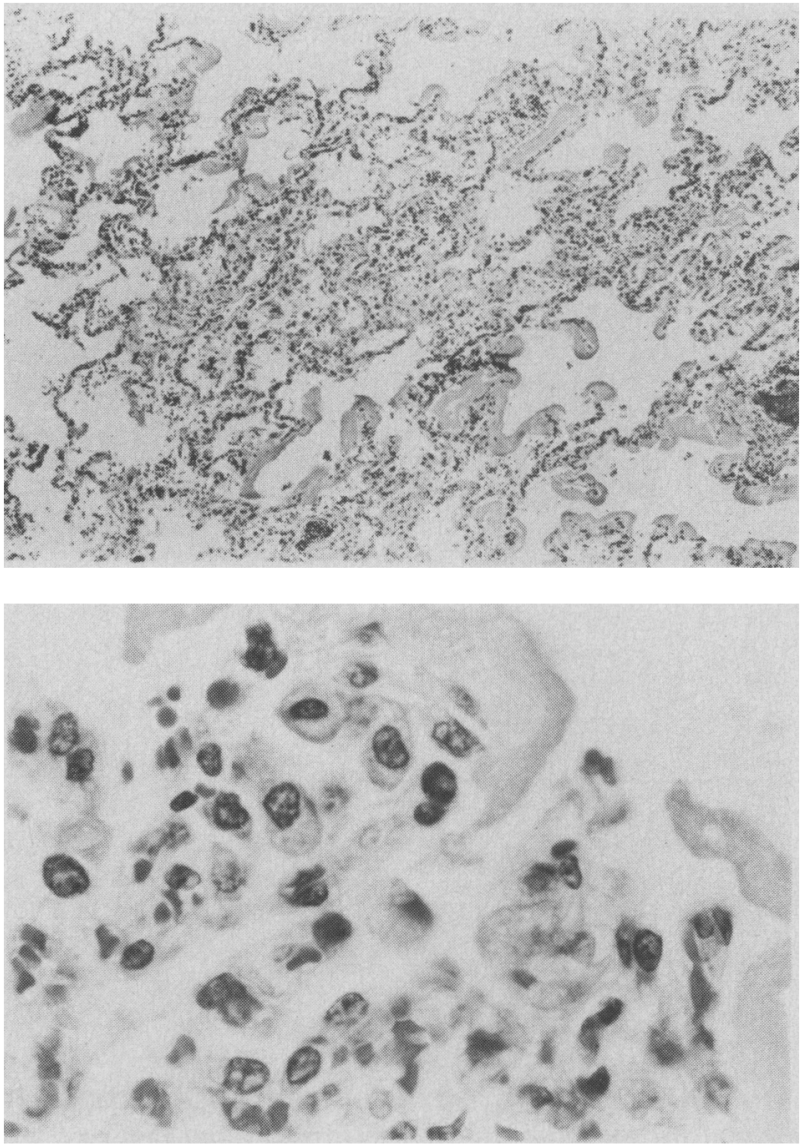

Figure 2. Top, lung tissue from Patient 1 demonstrating diffuse alveolar injury with intra-alveolar exudate and hyaline membranes (hematoxylin and eosin stain; original magnification $\times 80$, reduced by 50 percent). Botfom, detailed view of a representative area of lung tissue from Patient 1 demonstrating mononuclear cells without detectable neutrophils (hematoxylin and eosin stain; original magnification $\times 800$, reduced by 50 percent).

$\mathrm{C}$, Adriamycin, and 5-fluorouracil. Because of persistent vomiting, he was hospitalized and found to be neutropenic. At the time of admission, he had no respiratory complaints. On the fifth hospital day, he experienced chills, fever, and hypotension. Culture of blood samples obtained at the time showed group $D$ enterococci. Soon thereafter, he became dyspneic, and within 30 hours, he required intubation because of progressive respiratory failure. Chest roentgenography demonstrated diffuse, bilateral alveolar infiltrates. Measurement of arterial blood gases immediately after intubation revealed an oxygen tension of $93 \mathrm{~mm} \mathrm{Hg}$, carbon dioxide tension of $34 \mathrm{~mm}$ $\mathrm{Hg}$, and a pH of 7.31, while the patient was breathing 100 percent oxygen. His static total respiratory system compliance was $25 \mathrm{ml} / \mathrm{cm} \mathrm{H}_{2} \mathrm{O}$. Despite intensive therapy, he died on the eighth hospital day. As demonstrated in Figure
1 , the onset of neutropenia preceded by at least several days the first evidence of the adult respiratory distress syndrome.

We considered the possibility that neutrophils may have been sequestered within the lungs of the three neutropenic patients with the adult respiratory distress syndrome, despite the greatly decreased numbers of neutrophils within their peripheral blood. To investigate this possibility, we examined tissue specimens obtained from two of these patients who died and underwent postmortem examination. Patient 1 was just described. Patient 2 was a renal transplant recipient with azathioprine-induced neutropenia in whom respiratory distress developed in association with Escherichia coli septicemia. Multiple areas of the lungs from each of the two patients were sampled for histologic study. In both patients, many of the alveolar septae were covered with membranes composed of fibrin and cellular debris (Figure 2). Interstitial
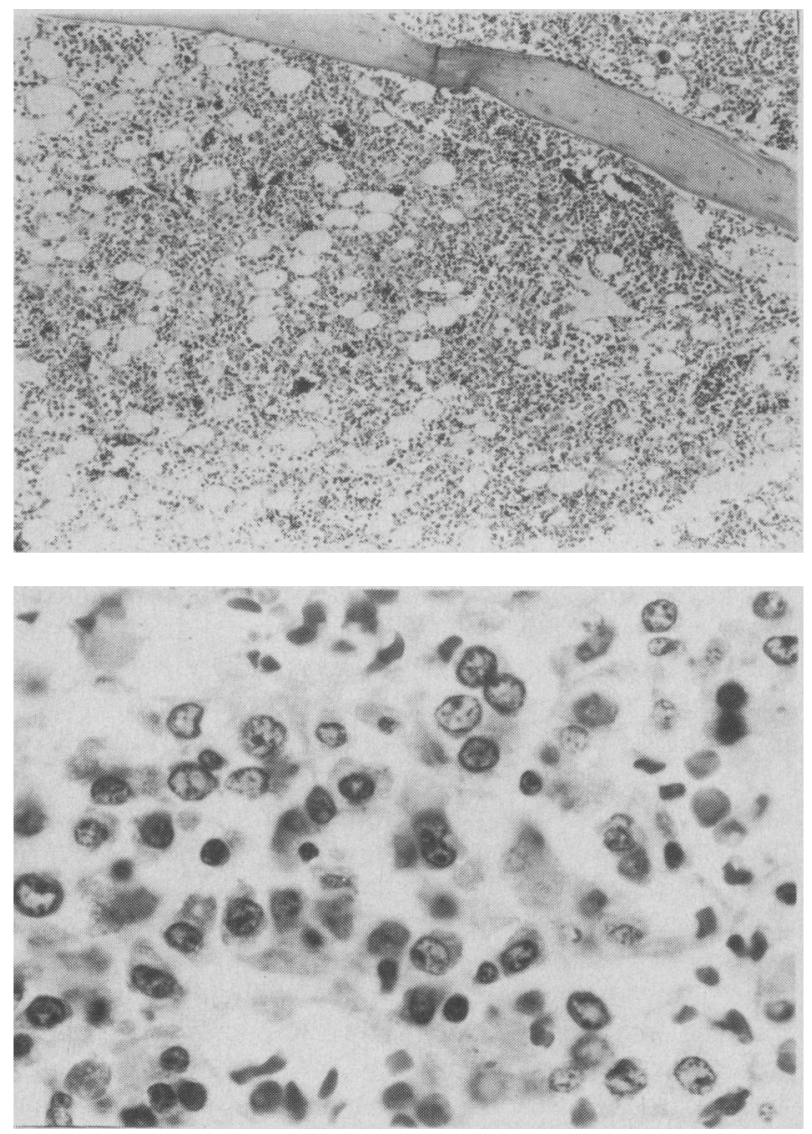

Figure 3. Top, bone marrow from Patient 1 (hematoxylin and eosin stain; original magnification $\times 80$, reduced by 50 percent). Bottom, detailed view of a representative area of bone marrow from Patient 1 demonstrating the absence of maturing neutrophils (hematoxylin and eosin stain; original magnification $\times 800$, reduced by 50 percent). 
edema and regenerative pneumatocytes were present. Although occasional monocytes and lymphocytes were evident, no neutrophils were detected. Examination of bone marrow from both patients revealed the absence of maturing neutrophils (Figure 3 ).

\section{COMMENTS}

In 1967, the term "respiratory distress syndrome in adults" was first applied to the acute development of noncardiogenic pulmonary edema and respiratory failure in association with a variety of nonpulmonary diagnoses [1]. Since that initial description, a large number of diseases have been associated with the development of the adult respiratory distress syndrome. Grouping these conditions together into a single syndrome has proved valuable, particularly because it emphasizes that the patients share distinctive physiologic abnormalities. Also, the recognition that a patient fulfills the criteria for the adult respiratory distress syndrome has important therapeutic implications regarding ventilator and fluid management [16].

Evidence from human and animal studies has suggested that phagocytic neutrophils are involved in the pathogenesis of the respiratory distress syndrome [2]. In many animal models of the syndrome, neutrophil depletion protects the lung from injury [8-12]. In our study, however, we found that neutropenia in humans does not totally prevent the development of the adult respiratory distress syndrome. Thus, for the neutropenic patients, other etiologic agents must have been operative. The list of possible candidates includes macrophages, platelets, clotting factors, kinins, and other inflammatory mediators [17].

We were not able to examine the lung tissue of our patients precisely at the onset of the adult respiratory distress syndrome. However, we believe that information available for Patient 1 makes it unlikely that neutrophils were present in significant numbers early in his course. In particular, the patient had severe chemotherapy-induced neutropenia that began at least several days prior to the development of respiratory distress. At the time of death, not only were his lungs devoid of detectable neutrophils, but his bone inarrow, the source of neutrophils, was also depleted.

The occurrence of the respiratory distress syndrome in our three neutropenic patients does not preclude the pussibility that neutrophils were important in some, if not all, of the remaining 15 patients. There is reason to believe that many distinct pathogenic mechanisms can lead to the syndrome. For example, acute noncardiogenic pulmonary edema would be expected to occur from insults directed at capillary endothelium, basement membranes, alveolar epithelium, or alveolar surfactant $[18,19]$. There is no a priori reason to suspect that there has to be a single damaging agent, e.g. the neutrophil. In fact, among the various animal models of acute respiratory distress, some require the presence of neutrophils, and others do not $[2,20]$.

In summary, neutropenia did not totally protect the lungs of bacteremic patients from the development of the adult respiratory distress syndrome. in three neutropenic patients with the syndrome, other etiologic agents must have been responsible. The adult respiratory distress syndrome is likely to be a multifactorial syndrome, possibly with both neutrophil-dependent and neutrophil-independent pathogenic mechanisms. In the appropriate clinical setting, the diagnosis of the adult respiratory distress syndrome should not be excluded solely because the patient is neutropenic.

\section{REFERENCES}

1. Ashbaugh DG, Bigelow DB, Petty TL, Levine BE: Acute respiratory distress in adults. Lancet 1967; 1l: 319-323.

2. Tate RM, Ropine JE: Neutrophils and the adult respiratory distress syndrome. Am Rev Respir Dis 1983; 128: 552-559.

3. Bachofen M, Weibel ER: Alterations of the gas exchange apparatus in adult respiratory insufficiency associated with septicemia. Am Rev Respir Dis 1977; 116: 589-615.

4. Lee CT, Fein AM, Lippmann M, Holtzman H, Kimbel P, Weinbaum G: Elastolytic activity in pulmonary lavage fluid from patients with adult respiratory distress syndrome. $\mathrm{N}$ Engl J Med 1981; 304: 192-196.

5. McGuire WM, Spragg RG, Cohen AB: Studies on the pathogenesis of the adult respiratory distress syndrome. J Clin Invest 1982; 69: 543-553.

6. Zimmerman GA, Renzetti AD, Hill HR: Functional and metabolic activity of granulocytes from patients with the adult respiratory distress syndrome. Am Rev Respir Dis 1983; 127: $290-300$.
7. Weiss SJ, LoBuglio AF: Biology of disease. Phagocyticgenerated oxygen metabolites and celiular injury. Lab invest 1982; 47:5-18.

8. Johnson KJ, Ward PA: Role of oxygen metabolites in immune complex injury of lung. J Immunol 1981; 126: 2365-2369.

9. Till GO, Johnson KJ, Kunkel R, Ward PA: Intravascular activation of complement and acute lung irjury. Dependency on neutrophils and toxic oxygon metabolites. J Clin Invest 1982; 69: 1126-1135.

10. Hohn DC, Meyers AJ, Gherini ST, Bechman A, Markisa RE, Churg AM: Production of acute pulmonary injury by leukocytes and activated complement. Surgery 1980; 88 : $48-57$.

11. Hosea $S$, Brown E, Hammer C, Frank M: Role of complement activation in a model of adult respiratory distress syndrome. J Clin Invest 1980; 66: 375 382 .

12. Flick MR, Porel A, Staub NC: Leukocytes are required for increased lung microvascular permeability after mi- 
croembolization in sheep. Circ Res 1981; 48: 344-351.

13. Armitage P: Statistical methods in medical research. New York: J Wiley and Sons, $1971 ; 116-118,135-138$.

14. Mood AM, Graybill FA, Boes DC: Introduction to the theory of statistics. New York: McGraw-Hill, 1974; 389-393.

15. Thommasen HV, Russel JA, Boyko WJ, Hogg JC: Transient leucopenia associated with adult respiratory distress syndrome. Lancet 1984; I: 809-812.

16. Bone RC: Treatment of severe hypoxemia due to the adult respiratory distress syndrome. Arch Intern Med 1980; 140: 85-89.

17. Rinaldo J, Rogers RM: Adult respiratory distress syndrome: changing concepts of lung injury and repair. $N$ Engl $J$ Med 1982; 306: 900-909.

18. Crandall ED, Staub NC, Goldberg HS, Effros RM: Recent developments in pulmonary edema. Ann Intern Med 1983; 99: 808-822.

19. Guyton AC, Moffatt DS: Role of surface tension and surfactant in the transepithelial movement of fluid and in the development of pulmonary edema. Prog Respir Res 1981; 15: $62-75$.

20. Fairman RP, Glauser FL, Falls R: Increases in lung lymph and albumin clearance with ethchlorvynol. J Appl Physiol 1981; 50: 1151-1155. 